\title{
Symbiotic Properties of Rhizobial Isolates of Manang and Kathmandu Districts
}

\author{
Bikash Baral $^{1}$ and Vimal Narayan Gupta ${ }^{2}$ \\ ${ }^{1}$ Nepal Academy of Science and Technology, Khumaltar, Lalitpur \\ ${ }^{2}$ Central Department of Botany, Tribhuvan University, Kirtipur, Kathmandu \\ e-mail:bikubaral@yahoo.com
}

\begin{abstract}
Nitrogen is a major limiting factor for primary production in any ecosystem and also the vital component of nucleic acids, proteins, chlorophylls and many other metabolic molecules. Legume-Rhizobium symbiotic system is known to be an efficient converter of ambient nitrogen into its nutrient form that increases soil fertility. The present work involved isolation, characterization and authentication of two rhizobial isolates, KR1 and MR2 obtained by trap method using broad bean (Vicia faba L.) from the field soils of Kathmandu and upper Manang (trans-himalayan region). Their culture characters and symbiotic effectiveness were analysed using standard methods. $V$. faba was used to study their effectiveness on various parameters such as plant height, roots length, shoot length, nodule number, nodules biomass, plant biomass, content of ureides in xylem sap, as indicative to fixed nitrogen and total nitrogen in nodules, stem and leaves were studied. Viable cell counts at $72 \mathrm{~h}$ of growth were $17.8 \times 10^{7}$ for MR2 and $14.1 \times 10^{7}$ for KR1. The isolates were similar for nodulation, plant biomass and nodule nitrogen (P $\left.\geq 0.05\right)$ the MR2 affected vigorous plant growth. However, the isolates showed significant differences $(\mathrm{P} \leq 0.05)$ with plant age on all the parameters studied. The kinetics of inoculation effects on nodulation, plant height, biomass, and total nitrogen were dealt with.
\end{abstract}

Key words: Rhizobium, rhizobial isolates, nodules, translocation

\section{Introduction}

The Rhizobium-legume symbiosis has continued to gain special attention in agricultural production in the past and is believed to be valued more in future as a potential option for mitigating the impacts of climate change in any ecosystem. The symbiotic nitrogen fixation has considerable advantage from an ecological as well as an economical point of view in the particular context of sustained soil fertility to support agricultural production or food security.

Of the estimated total nitrogen fixed worldwide in nature, a significant portion (87\% approx.) is the contribution of biological nitrogen fixation either from symbiotic associations (80\%) or from free living organisms (20\%)(Gutschick 1980, Vance et al. 1994). However, an effective Rhizobium-legume symbiosis is imperative to desirable crop production. The available nutrient nitrogen to plants has its source in both the biological nitrogen fixation (BNF) and applied fertilizers (Russelle \& Birr 2004).
In the context of Nepal, where most of the population (over 80\%) is depdends on agriculture, its relevance is high for the fertility status of cultivated soils which are low in nutrient nitrogen. Ever increasing population demands for more and more food production. Dependency of the subsistent farmers of Nepal on chemical fertilizers is risky for a sustained production since continued use of chemical fertilizers make the soil acidic. At present, when the farmers are venturing into commercial agriculture and organic farming practices, the importance of BNF is much more.

The present work focused on isolation of Rhizobium isolates from field soils of Manang (Himalayan habitat) and Kathmandu. They were tested for their efficiency of nitrogen fixation using broad bean ( $V$. faba L.) as a test plant. A comparative assessment of their effectiveness on different parameters was analysed. 


\section{Methodology}

Soil Source: Composite samples of cultivated field soils from upper Manang (Manang VDC fields located on the right side of the trail going to Khansar) and Kathmandu (Kirtipur west side) were obtained.

Trap Method: Trap method was used to isolate rhizobia from field soils. An amount of $500 \mathrm{~g}$ of a particular soil sample was placed in polythene bags (3"x6") to grow surface sterilized broad bean seeds. Healthy red-brown nodules were collected from some roots stored at $4 \pm 1^{\circ}$ C.

Rhizobial Isolation: Sterilized nodules were cut transversely by means of a sterilized blade. A loopful of the inner core of nodules, i.e. bacteroids were streaked on yeast extract mannitol agar (YMA)-plates. One plate was inoculated with one nodule bacteroid. The inoculated plates were incubated at $28 \pm 1^{\circ} \mathrm{C}$. Concave colonies, with translucent, shiny, milky, with entire margin, were obtained after 5 days of incubation.

Pure Culture: Individual colony was transferred to fresh YMA-plates for reconstitution and thereby pure culture isolates were obtained. Thus, the isolate with origin in the soil sample of Kirtipur (Kathmandu) was taken as KR1 and corresponding isolate of Manang was MR2.

Authentication: Confirmatory test for a bacterium to be Rhizobium is plant infection and induction of nodule on the roots of test legume. Pouch test method was used for this purpose. $V$. faba. was used as the test legume. Experiments on plant infection test, Gram Staining, Acid production behaviour and viable cells counts (mean generation time) were carried out using standard methods as described by Somasegaran and Hoben (1994).

Experimental settings: Surface sterilized broad bean seeds were sown into earthen pots (6"x9") (3 per pot to thin to one). Heat sterilized river sand was used as the medium of plant growth. Nitrogen free or complete nutrient solution was added to the pots as recommended (Somasegaran \& Hoben 1994). Sterilized tap water was used to irrigate the pots when required. Plants were raised in green house. The plants were harvested every 10 days after 20 days of sowing. Different parameters like nodule induction shoot length, nodules biomass, shoots biomass, total $\mathrm{N}$ content of leaf, shoot and nodules were carried out starting from 20 days at every 10 days ending at 40 days. Total $\mathrm{N}$ of milled dry plant tissue was determined by Kjeldahl digestion followed by measurement of $\mathrm{NH}_{4}^{+}$using the colorimetric method (Young et al. 1942).

Experimental design and data analysis: Treatments $=4$, Rhizobial isolates $=2$, Combinations $=4 \times 2=8 \times$ Replications 4= 32 units. Experiments were repeated 2 times. Average data were used for ANOVA. LSD was analysed for significant cases only. Bacterial inoculum was designated with ' $\mathrm{I}$ ' and nitrogen as ' $N$ '. The presence was indicated with '+' sign and absence with '-' sign.

\section{Results}

Authentication: Bacterial colonies of both isolates KR1 and MR2 were creamy white to translucent, smooth, circular and elevated. They did not absorb congo red confirming them to be the rhizobial colonies.

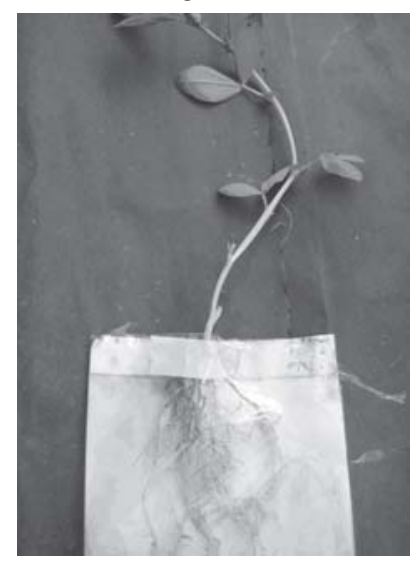

Fig.1. Plant infection test in pouch

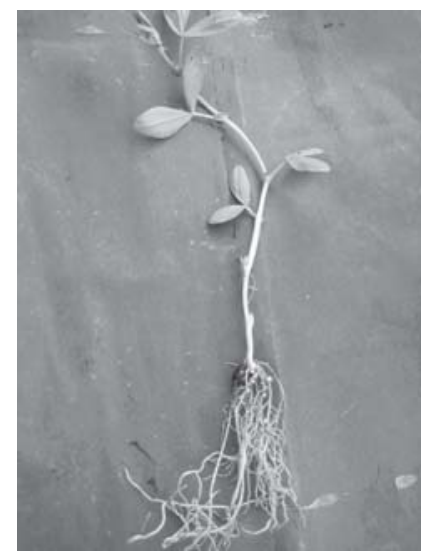

Fig.2. Nodules induced by inoculated isolate 
The blue colour of BTB changed to yellow that indicated the isolates were acid producing. None of the isolates took violet colour of crystal violet. They looked pink and rod shaped when observed under high power microscope. Both of the isolates induced nodules in plastic pouches. These attributes suggested them to be Rhizobium.

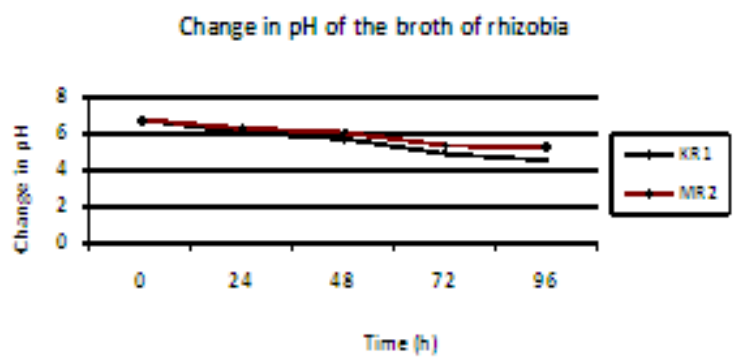

Fig.3. Change in $\mathrm{pH}$ of the broth at different time intervals

The change in $\mathrm{pH}$ of the broth was observed throughout the experiment and second rhizobial isolate (MR2) was found to be more acid producing than first isolate (KR1).

\section{Mean generation time}

Counting of the rhizobial population at different time intervals were carried out simultaneously by drop plate and pour-plate methods. The mean generation time

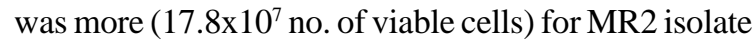

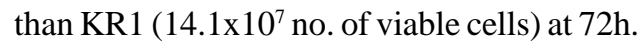

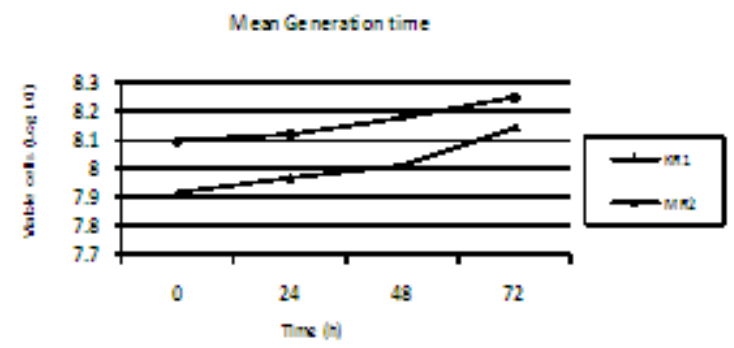

Fig.4. Multiplication of bacterial cells in different time intervals

\section{Nodules induction}

Counting of the nodules was done after 20 days of plant age and the different treatment combinations gave different results for both the isolates. Plant samples belonging to treatments I-N, I-N and I $+\mathrm{N}$ were found profusely nodulated over -I-N i.e. uninoculated as observed at 20, 30 and 40 days of plant age. The effect was significant $(\mathrm{P}<0.05)$ for both of the rhizobial isolates KR1 and MR2 for nodulation.

$$
\text { Effect of KR1 and Mr } 2 \text { onnumber of nodules }
$$

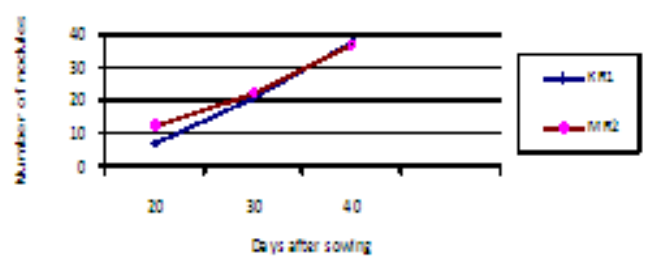

Fig 5. Effect of KR1 and MR2 on number of nodules

The treatment combinations I-N, I-N and I+N showed maximum number of nodules (nodules induction) when observed at 20, 30 and 40 days of plant age respectively. Although both of the rhizobial isolates, KR1 and MR2 affected nodulation significantly with age of the plants $(\mathrm{P}<0.05)$ they behaved similar $(\mathrm{P}>0.05)$.

The inoculum of the second rhizobial isolate was found to have more effect on the shoot length then that of the first rhizobial isolate (treatments: 4.462, bacterial blocks: $12.573, \mathrm{P}<0.05$ and LSD: 4.566). The increment in the length of the root was observed on the test plants which revealed insignificant difference (treatments: 1.667, bacterial blocks: 26.102, $\mathrm{P}>0.05$ and LSD: $8.0 \mathrm{~cm})$.

The sequences of inoculums effects of KR1 and MR2 at 40 days after seed sowing (DAS) was found in the order $(\mathrm{I}+\mathrm{N})>(\mathrm{I}-\mathrm{N})>(\mathrm{O}+\mathrm{N})>(\mathrm{O}-\mathrm{O})$ and the ANOVA showed the insignificant difference in treatment combinations and the blocks (treatments: 1.98, blocks: 0.934, P>0.0 5 and LSD: 0.479g). The result for the shoot biomass showed the insignificant difference at 95\% level of significance (treatments: 0.493 , bacterial blocks: $1.41, \mathrm{P}>0.05$ and LSD: $0.25 \%$ ), for stem $\mathrm{N}$ (treatments: 1.328, bacterial blocks: 0.859, P $>0.05$ and LSD: $0.202 \%$ ) and for leaves N (treatments: 4.028, bacterial blocks $0.864, \mathrm{P}<0.05$ and LSD: $0.311 \%$ ).

Data on plant biomass produced under inoculated and uninoculated as well as nitrogen fertilizer (urea) used and not used (control) treatment conditions were used to calculate the symbiotic effectiveness of both the 
rhizobial isolates, but showed no significant difference $(\mathrm{P}>0.05)$ on rhizobial inoculation and fertilizer nitrogen application. However, plant age significantly affected the symbiotic effectiveness reaching the maximum at $4^{\text {th }}$ week of plant growth.
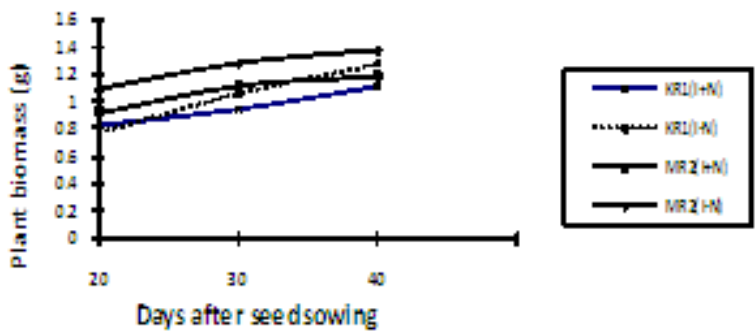

Fig.6. Plant biomass (g) inoculated with KR1 and MR2

\section{Total le af nitrogen (\%) of KR1 and MR2}

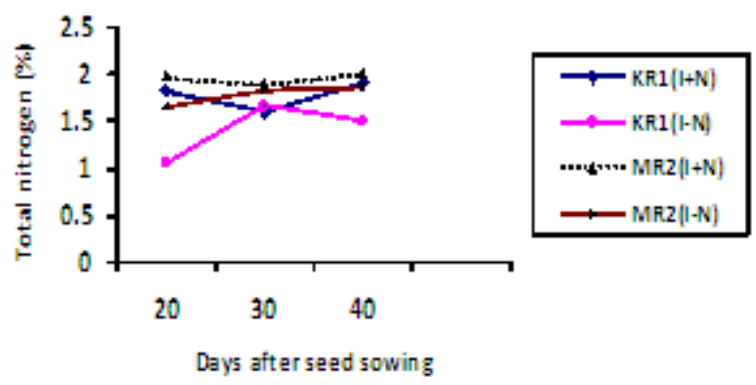

Fig.7. Total leaf nitrogen (\%) of plants inoculated with KR1 and MR2

Total stem N (\%) of KR1 and MRz

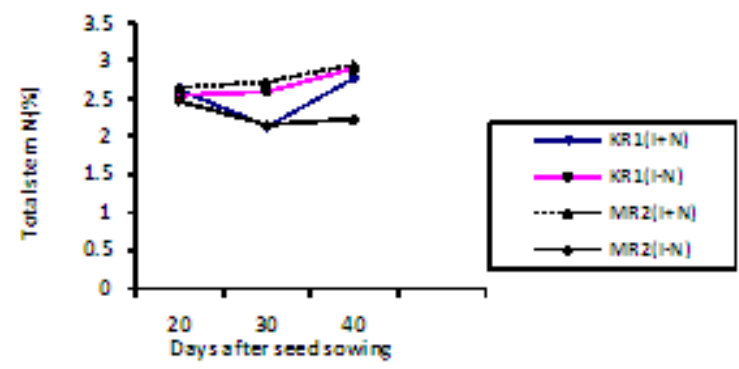

Fig.8. Total stems nitrogen (\%) of plants inoculated with KR1 and MR2
Total Nodules N (\%) of KR1 and MR2

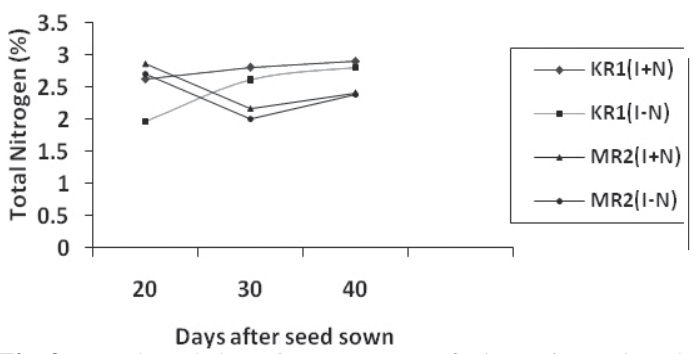

Fig.9. Total nodules nitrogen (\%) of plants inoculated with KR1 and MR2

\section{Discussion}

Legume inoculation has dual objectives, to induce nodulation promptly and effectively in the inoculated crop and to establish populations of the inoculant organism in the soil which are sufficiently large and persistent to provide a potent source of inoculum for subsequent crops. The symbiotic effectiveness is the reflection of the effective symbiotic association of inoculated rhizobia in a given soil field condition.

The soil $\mathrm{pH}$ improves the symbiotic potentialities of inoculant rhizobia. Selection of rhizobial strain for the inoculum production is based upon the ability of the strain in question to form effective nodules on the roots of legume for which it is recommended and ultimately enhance the yield of plants under a wide range of soil and climatic conditions (Burton 1979, Singleton et al. 1982). Plants metabolisms always tend to have a fixed $\mathrm{C}$ : $\mathrm{N}$ ratio and when the $\mathrm{N}$ input is increased in the plant, the equilibrium shifts towards the accumulation of more of the biomass via vigorous growth of the plant.

The nitrogen fixation process can be influenced by various factors viz. plant genotype and age, plant and rhizobia interactions, change in soil physiochemical conditions and various management practices. Some of the common approaches to enhance the biological nitrogen fixations may be the inoculation with proven strains, microbial screening for improved strains, hostplant screening and breeding and adoption of cropping system with good cultural practices.Plants with inoculum had a good deal of effective nodules on their root systems at 40 days after seed sowing 
(DAS). Visible nodules appeared at 20 DAS. The delay in nodulation might be due to exposure of sunlight and continuous cloud during the initial period of the experiment. The low light exposure leads into the low synthesis of photosynthetas (carbohydrates). The carbohydrates supplied by host plants used by bacteroids as a skeleton for incorporation of $\mathrm{NH}_{3}$ and derive energy through respiration which is utilized in nitrogen fixation and also run their own metabolism.

Statistical analyses were performed for nodulation, shoot length, shoot dry matter and $\mathrm{N}$ content for all the experimental sets. According to Somasegaran and Hoben (1994), shoot dry weight is the most reliable parameter and is routinely used as an indicator or relative stain effectiveness. The correlation output revealed the positive correlation between nodule number and the growth parameters. The test plant accumulated higher biomass with the inoculums of second isolate and is positively correlated with nodule biomass, numbers and nitrogen content at 40 DAS.

The calculation of the F-test between the rhizobial isolates also showed the significant differences on each of the experiments. Some of the slight deviation from the normal path is due to the unequal light distribution, variability of the nutrients available, soil $\mathrm{pH}$ and other inorganic compounds present in the soil.

\section{Acknowledgements}

The authors would like to acknowledge all those helping hands especially Bimal Kunwar, Sishir Panthi, Madhav Pandey, Govinda Joshi, Bijaya Laxmi Maharjan, Pushpa Sharma, Trilok Nath Thakur and Deepak Mahat.

\section{References}

Burton, J.C. 1979. Rhizobium species. In: Microbial technology, $2^{\text {nd }}$ ed. Vol I. (Ed. H.J.Peppler, and Perlman). Academic Press, New York. pp. 29-58.

Gutschick, V.P. 1980. Energy flow in the nitrogen cycle, especially in fixation. In: W.E. Newton, WH, Nitrogen Fixation. Vol I. Orme Johnson. University Park Press, Baltimore, USA. pp. 17-27.

Russelle, M.P. and A.S. Birr. 2004. Large-scale assessment of symbiotic dinitrogen fixation by crops: Soybean and alfalfa in the Mississippi river basin. American Society of Agronomy 96:1754-1760.

Singleton, P.W., A.E. Swaify. and B.B. Bahlool. 1982. Effect of salinity on Rhizobium growth and survival. Applied and Environmental Microbiology 44: 884-890.

Somasegaran, P. and J. Hoben. 1994. Handbook for rhizobia: Methods in legume-Rhizobium Technology. Springerverlag, New York p450.

Vance, C.P., R.C. Gregerson., D.L. Robinson., S.S. Miller and J.S. Gantt. 1994. Primary assimilation of nitrogen in alfalfa nodules-molecular-features of the enzymes involved. Plant Sci. 101:51-64.

Vincent, J.M. 1970. A manual for the practical study of the root nodule bacteria. IBP Handbook No. 15. International Biological Programme, Blackwell Scientific Publications, Oxford.

Young, E.G. and C.F.Conway.1942. On the estimation of allantoin by the Rimini-Schryver reaction. J. Biol. Chem. 142:839-853. 
Nepal Journal of Science and Technology 11 (2010) 95-100 